\title{
Experimental Results and Bifurcation Analysis on Scaled Feedback Control for Subsonic Cavity Flows
}

\author{
X. Yuan, E. Caraballo, M. Debiasi, J. Little, A. Serrani*, H. Özbay and M. Samimy
}

\begin{abstract}
In this paper, we present the latest results of our ongoing research activities in the development of reducedorder models based feedback control of subsonic cavity flows. The model was developed using the Proper Orthogonal Decomposition of Particle Image Velocimetry images in conjunction with the Galerkin projection of the Navier-Stokes equations onto the resulting spatial eigenfunctions. Stochastic Estimation method was used to obtain the state estimation of the Galerkin system from real time surface pressure measurements. A linear-quadratic optimal controller was designed to reduce cavity flow resonance and tested in the experiments. Realtime implementation shows a significant reduction of the sound pressure level within the cavity, with a remarkable attenuation of the resonant tone and a redistribution of the energy into various modes with lower energy levels. A mathematical analysis of the performance of the $L Q$ control, in agreement with the experimental results, is presented and discussed.
\end{abstract}

\section{INTRODUCTION}

The generation of tones by flow past an open cavity is a well known phenomenon which affects landing gear and weapons bays on aircraft. This flow is characterized by a complex feedback process that leads to self-sustained oscillations referred to as Rossiter [13] mechanism: small disturbances are amplified by the cavity shear layer, and produce acoustic waves when they impinge on the downstream corner of the cavity; these acoustic waves then propagate upstream and excite further instabilities in the shear layer, leading to self-forcing. The sound pressure levels of the tones can be as high as $160 \mathrm{~dB}$, which can cause structural damage in air vehicles. While passive and openloop active control methodologies were extensively applied for the suppression of cavity flow tones in past studies, feedback control has only recently been employed to this problem (see Cattafesta et al. [2] for a recent review) and the effects of the closed-loop dynamic control on the flow dynamics are not well understood yet.

The flow control team at the Ohio State University (OSU) Collaborative Center of Control Science (CCCS) is working to develop tools and methodologies for closed-loop aerodynamic flow control. The team, composed of researchers

This work is supported in part by AFOSR and AFRL/VA through the Collaborative Center of Control Science (Contract F33615-01-2-3154).

$X$. Yuan and A. Serrani are with the Department of Electrical \& Computer Engineering, the Ohio State University, Columbus, OH, 43210

E. Caraballo, M. Debiasi, J. Little and M. Samimy are with Gas Dynamics and Turbulence Laboratory, Mechanical Engineering Department, the Ohio State University, Columbus, OH 45235-7531.

H. Ozbay is with the Department of Electrical \& Electronies Engineering, Bilkent University, Ankara, TR-06533, Turkey; on leave from The Ohio State University.

*Author for correspondence. Email: serrani $(a)$ ece.osu.edu from Departments of Mechanical Engineering and Electrical and Computer Engineering at OSU, Air Force Research Laboratory, and NASA Glenn, possesses synergistic capabilities in all of the required multidisciplinary areas of flow simulation, reduced order modeling, controller design, and experimental integration and implementation of the components along with actuators and sensors,. The ultimate goal is to enable the use of closed-loop aerodynamic flow control to control the flow over maneuvering air vehicles and ultimately to control the motion of the vehicles themselves. The initial application chosen as a benchmark problem for study is closed-loop control of the acoustic resonance of a flow over a shallow cavity [14], [15], [18]. The objective of the current work is to provide a comprehensive overview of the activity of the group on modeling and feedback control design, including a presentation of the specific experimental setup and a mathematical discussion of the results obtained in experiment and simulation.

The paper is organized as follows: the experimental apparatus is described in Section II. Section III discusses reduced-order modeling techniques of cavity flow dynamics. Controller design, real time implementations and analysis on the results are presented in Section IV, followed by concluding remarks in Section $\mathrm{V}$.

\section{EXPERIMENTAL APPARATUS}

In this section we outline the experimental setup described in more detail in Debiasi and Samimy [6]. The core of the experimental setup consists of an optically accessible, blow-down type wind tunnel with a test section of width $W=$ height $H=50.8 \mathrm{~mm}$. A cavity that spans the entire width of the test section is recessed in the floor with a depth $D=12.7 \mathrm{~mm}$ and length $L=50.8 \mathrm{~mm}$ for an aspect ratio $L / D=4$. For control the cavity shear-layer receptivity region is forced by a 2-D synthetic-jet type actuator issuing at 30 degrees relative to the main flow from a $1 \mathrm{~mm}$ slot embedded in the cavity leading edge and spanning the width of the cavity, see Fig. 1. A Selenium D3300Ti compression driver provides the mechanical oscillations necessary to create the zero net mass, nonzero net momentum flow for actuation. The actuator signals are produced by either a BK Precision 3011A function generator for open-loop forcing or by a dSPACE 1103 DSP control board in closed-loop studies and are amplified by a Crown D-150A amplifier. The pressure fluctuations are measured by Kulite dynamic pressure transducers placed in different locations in the test section. A dSPACE 1103 DSP board connected to a Dell Precision Workstation 650 
computer is used to simultaneously acquire the pressure signals at $50 \mathrm{kHz}$ through 16-bit channels and manipulates them to produce the desired control signal from a 14bit output channel. Each recording is band-pass filtered between 800 and $10,000 \mathrm{~Hz}$ to remove spurious frequency components. The "snapshots" of the flow field, required for

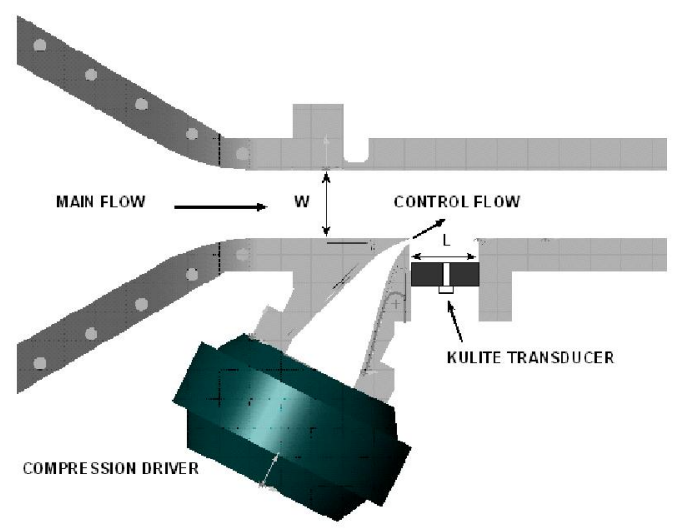

Fig. 1. Cutout of the wind tunnel showing the converging nozzle, the test section, the cavity, the actuator coupling, and the placement of a Kulite transducer in the cavity floor.

the development of the low dimensional model, are acquired and processed using a LaVision Inc. PIV system. Details of the PIV system, procedure, and results are presented in [11]. The main flow is seeded with Di-Ethyl-Hexyl-Sebacat (DEHS) particles by using a 4-jet atomizer upstream of the stagnation chamber. A dual-head Spectra Physics PIV-400 $\mathrm{Nd}$ :YAG laser operating at the 2nd harmonic $(532 \mathrm{~nm})$ is used in conjunction with spherical and cylindrical lenses to form a thin $(1 \mathrm{~mm})$, vertical sheet spanning the streamwise direction of the cavity at the middle of test section width. Two CCD cameras ( $2 \mathrm{~K}$ by $2 \mathrm{~K}$ ) with maximum acquisition frequency of $15 \mathrm{~Hz}$ capture the images when the laser is fired. Dedicated software is used to process the images and obtain the velocity flow field information. This setup gave a velocity vector grid of 128 by 128 over the measurement domain of $50.8 \mathrm{~mm}$ which translates to each velocity vector being separated by approximately $0.4 \mathrm{~mm}$. In the initial phase of the experiments only 2 velocity components are obtained.

\section{REDUCED-ORDER MODELING}

Reduced-order models of the flow were derived from PIV snapshots and surface pressure measurements of the cavity flow as described in detail by the authors in previous works [3], [4], [15]. The approach is based on the combination of three independent tools. First, the POD method is used to acquire a spatial basis of the velocity field. Then, Galerkin projection method is applied to obtain the flow model in the form of a set of ordinary differential equations for the POD temporal coefficients (states of the system model). In the process of projection, the domain of control input was separated from the rest of the flow field which results in a explicit expression of the control effect in the equations to facilitate the design of the feedback control law. Meanwhile stochastic estimation is used to estimate the states based on real time surface pressure measurements.

\section{A. POD Method}

The POD method was introduced to the fluid dynamics community by Lumley [12] as a way to extract large-scale structures in a turbulent flow. The general idea is to decompose the flow field into a set of orthogonal spatial basis that contains the most dominant characteristics of the flow. More details of the fundamentals of this method can be found in Holmes et al. [10]. The POD approach under investigation is the snapshot method [16], which is more suitable for highly spatially resolved data sets that can be obtained using numerical simulations or advanced laser-based flow diagnostics. Applying the POD method results in a temporal-spatial decomposition of a flow variable (e.g. the streamwise velocity $u(x, t))$ as:

$$
u(x, t) \cong \sum_{i=1}^{N} a_{i}(t) \varphi_{i}(x),
$$

where $\varphi_{i}(x)$ are spatial modes that capture the coherent structures and time coefficients $a_{i}(t)$ are function of time only and represent the time evolution of the corresponding coherent structures.

\section{B. Galerkin Projection}

The Galerkin projection method was used to acquire a low dimensional model of the cavity flow in the form of a set of ODE's for the time coefficients $a(t)$. The method relies on the projection of the governing equations of the flow, the compressible Navier-Stokes in this case, onto the POD spatial basis derived by POD decomposition. Refer to [3], [15] for a detailed description of the derivation of this model. It's worth to notice that a standard Galerkin projection results in an ODE model with external control effect implicitly expressed in the model, which is not useful for controller design. In order to derive a model where the control input appears explicitly in the equations, a control separation method was incorporated into the Galerkin projection procedure. The main idea of the control separation is to separate the sub-domain of space where the external control input is introduced into the flow field from the rest of the field, as detailed in Efe and Özbay [8], [9], which yields a system in the following form:

$$
\dot{a}=F+G a+\left(\begin{array}{c}
a^{T} H^{1} a \\
\vdots \\
a^{T} H^{N} a
\end{array}\right)+\left(B+\left(\begin{array}{c}
\bar{B}^{1} a \\
\vdots \\
\bar{B}^{N} a
\end{array}\right) \Gamma\right.
$$

where the matrices of constant coefficients $F, G, H^{i}, B$ and $\bar{B}^{i}, i=1, \ldots, N$, are obtained from the Galerkin projection, and $\Gamma(t)$ is the control input applied at the forcing location, detailed in Yuan et al. [19]. 


\section{Stochastic Estimation}

The Stochastic Estimation (SE) method has been used here to estimate the time coefficient of the Galerkin system (2) for feedback flow control. SE was originally proposed and used by Adrian [1] as a method to extract coherent structures from a turbulent flow field. The technique estimates flow variables at any location by using statistical information about the flow at a limited number of locations. In any realistic setting, the real-time experimental data could only be obtained via surface measurements (e.g. surface pressure or surface shear stress measurements). In the current work, quadratic stochastic estimation was employed to estimate the time coefficients of the flow model (2) directly from real-time measurements of surface pressures $p(t)$ at a small number of locations $L=6$. The estimates of the time coefficients can be written in the following form:

$$
\hat{a}_{i}(t)=C_{i k} p_{k}(t)+D_{i k l} p_{k}(t) p_{l}(t), \quad k, l=1, \ldots, 6
$$

where $C, D$ are the matrices of the estimation coefficients obtained by minimizing the average mean square error between the values of $a_{i}$ obtained from the snapshots and the estimated ones $\hat{a}_{i}$ at the same time.

\section{FEEDBACK CONTROL AND ANALYSIS}

In this section, we present the design of the model-based controller, discuss the real-time implementation results, and interpret the results from a mathematical perspective. The design procedure includes equilibrium computation, coordinates transformation, linear approximation and linearquadratic state feedback control design, which is described in [4], [19] in more detail. Three reduced-order flow models obtained by reduced-order modeling techniques discussed in Section III have been investigated in this work: (i) the baseline Mach 0.3 flow; (ii) the baseline flow forced at 3920 $\mathrm{Hz}$ using open-loop sinusoidal excitation; (iii) a flow model obtained from $P O D$ modes derived by concatenating PIV data sets from the two cases $\mathrm{i}$ and ii in time. The reducedorder flow model for all the cases for control design is the same nonlinear state space model given by (2), with $N=4$, whereas the numerical values of the model parameters obviously varies for each flow condition.

\section{A. Equilibrium Analysis and Model Simplification}

Defining $\widetilde{a}=a-a_{0}$ as the new set of coordinates, where $a_{0}$ is the equilibrium point computed for the model (2), and shifting the origin of the coordinates to the equilibrium point corresponding to the mean flow gives a simplified state space model in the new set of coordinates as

$$
\dot{\widetilde{a}}=\widetilde{G} \widetilde{a}+\left(\begin{array}{c}
\widetilde{a}^{\mathrm{T}} H^{1} \widetilde{a} \\
\vdots \\
\widetilde{a}^{\mathrm{T}} H^{4} \widetilde{a}
\end{array}\right)+\widetilde{B} \Gamma+\left(\begin{array}{c}
\bar{B}^{1} \widetilde{a} \\
\vdots \\
\bar{B}^{4} \widetilde{a}
\end{array}\right) \Gamma
$$

where

$$
\widetilde{G}=G+\left(\begin{array}{c}
\widetilde{a}_{0}^{T}\left(H^{1}+H^{1^{T}}\right) \\
\vdots \\
\widetilde{a}_{0}^{T}\left(H^{4}+H^{4^{T}}\right)
\end{array}\right), \widetilde{B}=B+\left(\begin{array}{c}
\bar{B}^{1^{T}} \\
\vdots \\
\bar{B}^{4^{T}}
\end{array}\right) a_{0} .
$$

Clearly, the modified model has an equilibrium point at the origin, which is more convenient for controller design and stability analysis.

\section{B. Linear Quadratic State Feedback Control}

A linear approximation of (4) at the origin is readily obtained as

$$
\dot{\widetilde{a}}=\widetilde{G} \widetilde{a}+\widetilde{B} \Gamma .
$$

The eigenvalues of the system matrix $\widetilde{G}$ have been computed for the three cases respectively and show the same qualitative features ( 2 unstable complex conjugate eigenvalues plus 2 stable real eigenvalues) and quantitative similarities as well. The presence of two unstable complex conjugate eigenvalues implies, as expected, that the mean flow (corresponding to the equilibrium) is an unstable solution for the Galerkin system (2).

Controllability of the pairs $(\widetilde{G}, \widetilde{B})$ for all cases and availability of real-time state estimates of the Galerkin model rendered by stochastic estimation (3) allow the use of linear state-feedback controller in this study. A standard linearquadratic (LQ) optimal controller based on the linearized model (5) was designed in the form

$$
\Gamma=-K \widetilde{a},
$$

and whereafter applied to the nonlinear Galerkin model. Simulation results have shown that the LQ controller designed for the linear approximation (5) succeeds in stabilizing the equilibrium of the four modes nonlinear Galerkin system (2), as indicated in Fig. 2.

\section{Real-Time Control Implementation}

In this part, we summarize the results obtained in the real time experimental implementation of the LQ feedback controller. It is important to point out that, to prevent damaging the actuator, the control input signal is limited to the range $\pm 10 \mathrm{~V}$. Since the LQ control by design is to completely eliminate the pressure fluctuations which results in quite large control gains, a constant saturation of the actuator was observed during closed-loop experiments. In order to keep the actuator below the saturation limits, a constant scaling factor $\alpha>0$ was introduced into the feedback loop. The largest feasible scaling factor has been found for all three flow models respectively as:

$$
\alpha_{1}=0.265, \quad \alpha_{2}=0.35, \quad \alpha_{3}=0.5,
$$

and the actual control law was scaled by $\alpha$ as:

$$
\Gamma_{\alpha}(t)=-\alpha K \hat{\tilde{a}}(t) .
$$

The scaled LQ controls (8) have also been simulated on corresponding nonlinear models (2) as depicted in Fig. 3. 

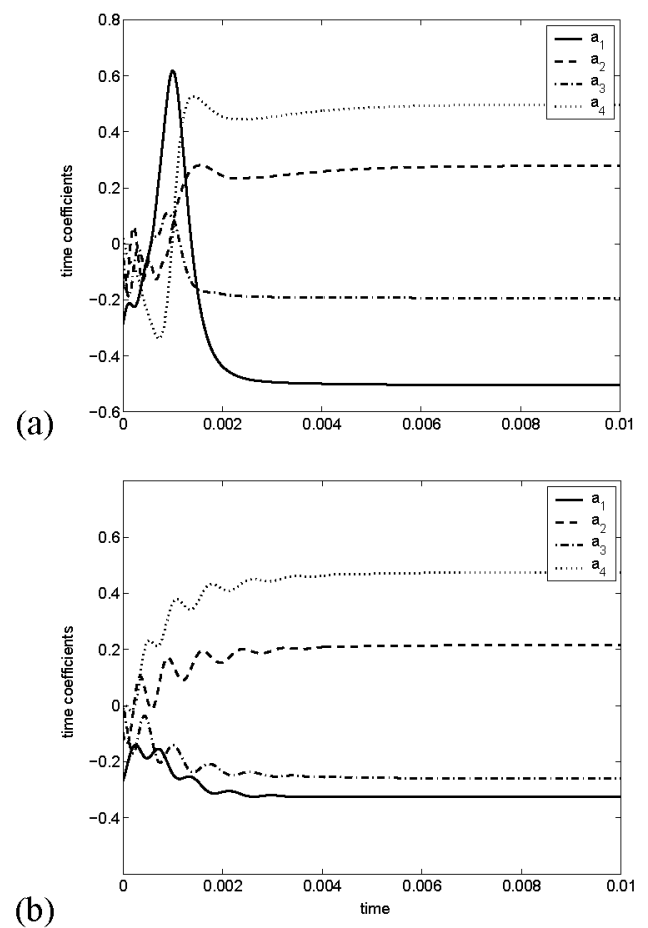

Fig. 2. Time coefficient solutions of the closed loop simulation results, where the plant is taken to be: (a) baseline flow model, (b) combined baseline and open-loop forced flow model (forcing frequency $3920 \mathrm{~Hz}$ ).

It is evident that, though the scaled LQ control for the given values of $\alpha$ is not able to asymptotically stabilize the origin of the nonlinear model (2), it nevertheless provides a significant reduction of the amplitude of the stable limit cycle in all three cases. This result is in agreement with a mathematical analysis carried on the nonlinear finitedimensional Galerkin model (4) given in Section IV-D.

The performance of the scaled control law (8) has been tested experimentally, for different flow conditions. Hereafter, we present the results obtained for Mach 0.3 cavity flow. Specifically, we present the closed-loop sound pressure level (SPL [dB]) recorded by the sensor located at central cavity floor with each scaled LQ controller designed on the basis of the three flow models discussed above. The results for closed-loop scaled LQ control, shown in Fig. 4 , show a considerable attenuation of the resonance peak, and a redistribution of the energy into various modes, especially lower frequency modes, with much lower energy level. The scaled LQ state feedback control has also been shown to successfully reduce the dominant Rossiter peak and provide good robustness when applied to off-design flow conditions around Mach 0.3.

\section{Mathematical Analysis of the Performance of $L Q$ Con- trol}

In what follows, we present a simple analysis, carried out on the basis of the nonlinear Galerkin model, to explain the closed-loop results obtained with the LQ feedback control.

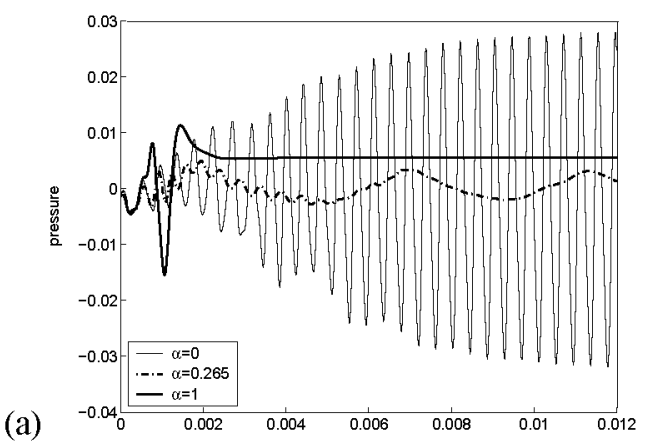

(b)

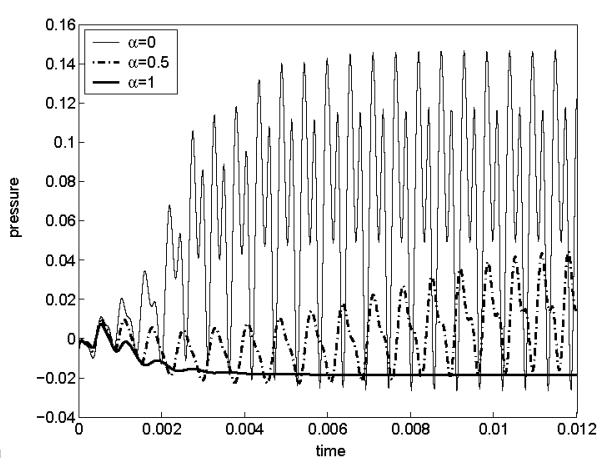

Fig. 3. Simulated closed loop response for the pressure fluctuation with different scaling factor. (a) baseline flow model (b) combined baseline and open-loop forced flow model (forcing frequency $3920 \mathrm{~Hz}$ ).

Recall that $\widetilde{G}$ in (4) has 2 unstable complex conjugate eigenvalues and 2 stable real eigenvalues. Let $T \in \mathbb{R}^{4 \times 4}$ be a nonsingular transformation that puts $\widetilde{G}$ in modal form

$$
\begin{gathered}
T \widetilde{G} T^{-1}=\left(\begin{array}{cc}
L_{1} & 0 \\
0 & L_{2}
\end{array}\right), \\
L_{1}=\left(\begin{array}{cc}
\sigma & -\omega \\
\omega & \sigma
\end{array}\right), \quad L_{2}=\left(\begin{array}{cc}
-\lambda_{1} & 0 \\
0 & -\lambda_{2}
\end{array}\right),
\end{gathered}
$$

where $\sigma>0, \omega>0, \lambda_{1}>0$, and $\lambda_{2}>0$. Partitioning the state vector according to the above decomposition, the Galerkin system is written in the new coordinates as

$$
\begin{aligned}
& \dot{\eta}=L_{1} \eta+M_{1} \Gamma+\psi_{1}(\eta, \zeta)+\gamma_{1}(\eta, \zeta) \Gamma \\
& \dot{\zeta}=L_{2} \zeta+M_{2} \Gamma+\psi_{2}(\eta, \zeta)+\gamma_{2}(\eta, \zeta) \Gamma
\end{aligned}
$$

where

$$
\begin{gathered}
T \widetilde{a}=\left(\begin{array}{l}
\eta \\
\zeta
\end{array}\right), \quad T \widetilde{B}=\left(\begin{array}{l}
M_{1} \\
M_{2}
\end{array}\right) \\
\psi_{i}(\eta, \zeta)=O\left(\|\eta\|^{2},\|\zeta\|^{2}\right), \\
\gamma_{i}(\eta, \zeta)=O(\|\eta\|,\|\zeta\|), \quad i=1,2 .
\end{gathered}
$$

Note that the control law $\Gamma=-\alpha K \widetilde{a}$ can be expressed in the new coordinates as

$$
\Gamma=-\alpha K_{1} \eta-\alpha K_{2} \zeta,
$$

for some matrices $K_{1}$ and $K_{2}$. Since it has been verified that the control input does not affect the location of the stable eigenvalues of the open-loop matrix $\widetilde{G}$, as it is typically 


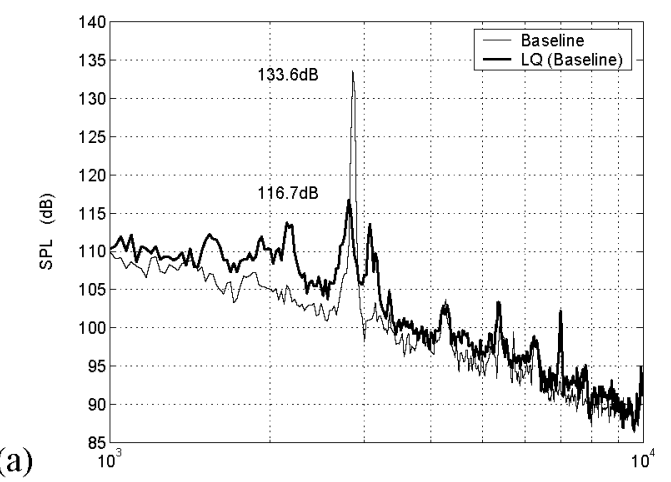

(a)

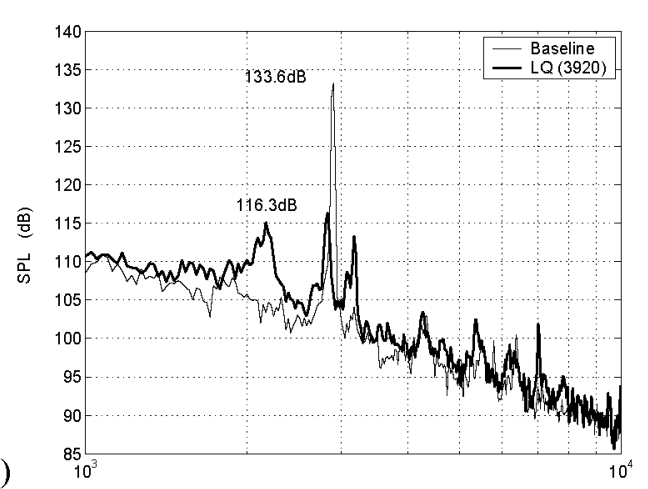

(b)

(c)

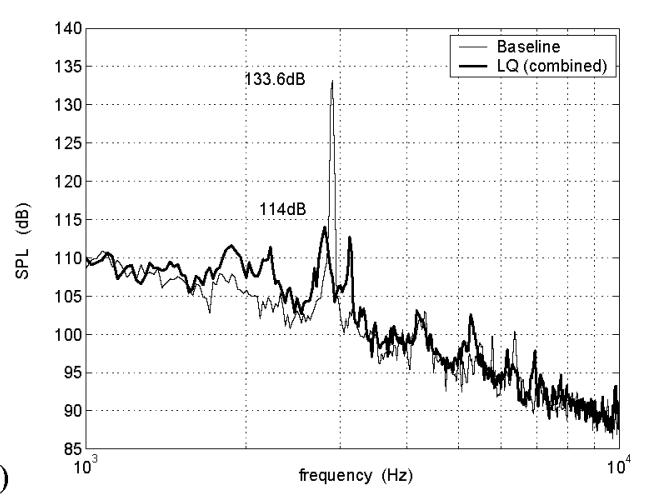

Fig. 4. Sound pressure level in closed-loop experiments with LQ design based on baseline flow model (a), forced flow model (b), and combined flow model (c).

the case for LQ-based design, necessarily $K_{2}=\left[\begin{array}{ll}0 & 0\end{array}\right]$. Therefore, the closed-loop system can be written in the form

$$
\begin{aligned}
& \dot{\eta}=\left(L_{1}-\alpha M_{1} K_{1}\right) \eta+\psi_{1}(\eta, \zeta)-\alpha \gamma_{1}(\eta, \zeta) K_{1} \eta \\
& \dot{\zeta}=-\alpha M_{2} K_{2} \eta+L_{2} \zeta+\psi_{2}(\eta, \zeta)-\alpha \gamma_{2}(\eta, \zeta) K_{1} \eta .
\end{aligned}
$$

An easy computation shows that the eigenvalues of the matrix $L_{1}-\alpha M_{1} K_{1}$ are given by

$$
\lambda\left(L_{1}-\alpha M_{1} K_{1}\right)=(1-2 \alpha) \sigma \pm j \sqrt{\omega^{2}+4 \alpha \sigma^{2}(1-\alpha)}
$$

and thus

$$
\lambda\left(L_{1}-\alpha M_{1} K_{1}\right) \approx(1-2 \alpha) \sigma \pm j \omega
$$

since $\omega>>\sigma$, and $\alpha \in[0,1]$. Letting $\mu=1-2 \alpha$, one obtains (modulo a unitary transformation)

$$
L_{1}-\alpha M_{1} K_{1}=\left(\begin{array}{cc}
\mu \sigma & -\omega \\
\omega & \mu \sigma
\end{array}\right) \text {, }
$$

and thus the spectrum of the closed-loop matrix

$$
\bar{L}(\mu)=\left(\begin{array}{cc}
L_{1}+(\mu / 2-1 / 2) M_{1} K_{1} & 0 \\
(\mu / 2-1 / 2) M_{2} K_{2} & L_{2}
\end{array}\right)
$$

splits into a pair of purely imaginary eigenvalues and a pair of negative real eigenvalues when $\mu=0$. This implies the existence of a center manifold for the trajectories of the Galerkin system. Specifically, denote for the sake of simplicity

$$
\begin{gathered}
\bar{L}(\mu)=\left(\begin{array}{cc}
\bar{L}_{11}(\mu) & 0 \\
\bar{L}_{21}(\mu) & L_{2}
\end{array}\right) \\
\phi_{i}(\eta, \zeta, \mu)=\psi_{i}(\eta, \zeta)-\alpha \gamma_{i}(\eta, \zeta) K_{1} \eta, \quad i=1,2
\end{gathered}
$$

and write the closed-loop Galerkin system as

$$
\begin{aligned}
\dot{\mu} & =0 \\
\dot{\eta} & =\bar{L}_{11}(\mu) \eta+\phi_{1}(\eta, \zeta, \mu) \\
\dot{\zeta} & =\bar{L}_{21}(\mu) \eta+L_{2} \zeta+\phi_{2}(\eta, \zeta, \mu)
\end{aligned}
$$

where

$$
\phi_{i}(\eta, \zeta, \mu)=O\left(\|\eta\|^{2},\|\zeta\|^{2}\right) \text { for all } \mu, \quad i=1,2
$$

and we have added a trivial dynamics for the bifurcation parameter $\mu$. The Center Manifold Theorem [5] establishes the existence of an exponentially attracting submanifold of the state space, which is described by the graph of a smooth mapping $\zeta=\pi(\eta, \mu)$ satisfying $\pi(0, \mu)=0$, $(\partial \pi / \partial \eta)(0, \mu)=0$, and

$$
\begin{aligned}
& \frac{\partial \pi}{\partial \eta}\left[\bar{L}_{11}(\mu) \eta+\phi_{1}(\eta, \pi(\eta, \mu), \mu)\right] \\
= & \bar{L}_{21}(\mu) \eta+L_{2} \pi(\eta, \mu)+\phi_{2}(\eta, \pi(\eta, \mu), \mu)
\end{aligned}
$$

for all $(\eta, \mu)$ in a neighborhood of $(0,0)$. This allows to reduce the analysis of the dynamics of the Galerkin system to its restriction onto the center manifold, which in the given set of coordinates reads as

$$
\left(\begin{array}{l}
\dot{\eta}_{1} \\
\dot{\eta}_{2}
\end{array}\right)=\left(\begin{array}{cc}
\mu \sigma & -\omega \\
\omega & \mu \sigma
\end{array}\right)\left(\begin{array}{l}
\eta_{1} \\
\eta_{2}
\end{array}\right)+\left(\begin{array}{l}
\phi_{11}(\eta, \pi(\eta, \mu), \mu) \\
\phi_{12}(\eta, \pi(\eta, \mu), \mu)
\end{array}\right) .
$$

A near-identity transformation into Poincarè normal form [17] yields

$$
\begin{aligned}
& \phi_{11}(\eta, \pi(\eta, \mu), \mu)=\left(-a(\mu) \eta_{1}-b(\mu) \eta_{2}\right) \rho^{2}+O\left(\|\eta\|^{5}\right) \\
& \phi_{12}(\eta, \pi(\eta, \mu), \mu)=\left(b(\mu) \eta_{1}-a(\mu) \eta_{2}\right) \rho^{2}+O\left(\|\eta\|^{5}\right),
\end{aligned}
$$

where $\rho=\sqrt{\eta_{1}^{2}+\eta_{2}^{2}}$ and $a(\mu)>0$. Using polar coordinates one obtains the system

$$
\begin{aligned}
& \dot{\rho}=\mu \sigma \rho-a(\mu) \rho^{3}+O\left(\rho^{5}\right) \\
& \dot{\theta}=\omega+b(\mu) \rho^{2}+O\left(\rho^{4}\right),
\end{aligned}
$$

where $\left.\theta=\tan ^{-1}\left(\eta_{2} / \eta_{1}\right)\right)$. The structure of the reduced system reveals that the original Galerkin system has a locally 
exponentially stable equilibrium at the origin for $\mu<0$, and undergoes a Hopf-Poincarè-Andropov bifurcation at $\mu=0$, with a stable limit cycle for $\mu>0$. The amplitude and frequency of the limit cycle are given respectively by

$$
\rho^{\star}=\sqrt{\frac{\mu \sigma}{a(\mu)}}, \quad \omega^{\star}=\omega+b(\mu) \frac{\mu \sigma}{a(\mu)}
$$

from which, since $a(\mu)=O(1)$, it is readily seen that the amplitude of the oscillation decreases as $\mu \rightarrow 0^{+}$. Recalling that $\mu=(1-2 \alpha)$, the results of the analysis can be summarized as follows:

1) If it is required to set $\alpha<0.5$ to avoid saturating the actuator, the origin of the Galerkin system can not be stabilized at all.

2) If this is the case, the application of linear feedback can still lower the amplitude of the limit cycle, but only up to the critical value imposed by the actuator limits.

The experimental results seem to support the results of the analysis, as linear feedback is capable to attenuate the resonance in the cavity to a certain extent, while complete suppression seems to be unattainable with the given actuation. However, it may still be possible to reduce the amplitude of the cavity tone beyond the limit achievable using linear feedback, resorting to different control strategies (nonlinear feedback or time-varying feedback). In particular, a viable strategy to be pursed is to increase $a(\mu)$, shaping the center manifold $\zeta=\pi(\eta, \mu)$ by means of nonlinear feedback.

\section{CONCLUSIONS AND FUTURE WORKS}

The work presented and discussed is part of our ongoing research activities in the development of reduced-order models based feedback control of subsonic cavity flows. PIV data and the POD technique are used to extract the most energetic flow features. The Galerkin projection of the Navier-Stokes equations onto the POD modes was used to derive a set of ordinary differential equations, which govern the time evolution of the modes, and to use for the controller design. Stochastic estimation is used to estimate the state of the Galerkin system from realtime pressure measurements. A linear-quadratic optimal controller was designed and implemented to reduce cavity flow resonance. A mathematical analysis was performed to explain the closed-loop results obtained with the LQ feedback control. Notwithstanding the encouraging results reported and discussed in this work, further investigation is needed to understand how to incorporate more effectively the presence of actuation in reduced-order POD models, and to pursue different control strategies (nonlinear feedback or time-varying feedback) to overcome the limit posed by linear feedback control.

\section{ACKNOWLEDGMENTS}

The support of AFRL/VA and AFOSR under Contract F33615-01-2-3154 is gratefully acknowledged. The authors would like to thank Drs. James Myatt, James DeBonis,
Chris Camphouse, John Casey and Kihwan Kim and Cosku Kasnakoglu for fruitful and insightful discussions.

\section{REFERENCES}

[1] R. J. Adrian, "On the Role of Conditional Averages in Turbulent Theory," Turbulence in Liquids, Science Press, Princeton, 1979.

[2] L. N. Cattafesta, D. R. Williams, C.W. Rowley and F. S. Alvi, "Review of Active Control of Flow-Induced Cavity Resonance", AIAA Paper 2003-3567, June 2003.

[3] E. Caraballo, J. Malone, M. Samimy, and J. DeBonis, "A Study of Subsonic Cavity Flows - Low Dimensional Modeling," AIAA Paper 2004-2124, June 2004.

[4] E. Caraballo, X. Yuan, J. Little, M. Debiasi, P. Yan, A. Serrani, J. Myatt and M. Samimy, "Feedback Control of Cavity Flow Using Experimental Based Reduced Order Model," AIAA Paper 2005-5269, June 2005.

[5] J. Carr, Applications of Centre Manifold Theory, Springer-Verlag, 1981.

[6] M. Debiasi and M. Samimy, "Logic-Based Active Control of Subsonic Cavity-Flow Resonance", AIAA Journal, Vol. 42, No. 9, pp. 1901-1909, September 2004.

[7] J. Delville, L. Cordier and J. P. Bonnet, "Large-Scale-Structure Identification and Control in Turbulent Shear Flows," In Flow Control: Fundamentals and Practice, (M. Gad-el-Hak, A. Pollard and J. Bonnet), pp. 199-273, Springer-Verlag, 1998.

[8] M. Ö. Efe and H. Özbay, "Proper Orthogonal Decomposition for Reduced Order Modeling: 2D Heat Flow," Proc. of IEEE Int. Conf. on Control Applications (CCA'2003), June 23-25, Istanbul, Turkey, $2003 a$.

[9] M. Ö. Efe and H. Özbay, "Integral Action Based Dirichlet Boundary Control of Burgers' Equation," Proc. of IEEE Int. Conf. on Control Applications (CCA'2003), June 23-25, Istanbul, Turkey, 2003b.

[10] P. Holmes, J. L. Lumley and G. Berkooz, Turbulence, Coherent Structures, Dynamical System, and Symmetry, Cambridge University Press, Cambridge, 1996.

[11] J. Little, M. Debiasi and M. Samimy, "Flow Structure in Controlled and Baseline Subsonic Cavity Flows", AIAA Paper 2006-0480, January 2006

[12] J. Lumley, "The Structure of Inhomogeneous Turbulent Flows", Atmospheric Turbulence and Wave Propagation, Nauka, Moscow, pp. 166-176, 1967

[13] J. E. Rossiter, "Wind Tunnel Experiments on the Flow Over Rectangular Cavities at Subsonic and Transonic Speeds", RAE Tech. Rep. 64037, 1964 and Aeronautical Research Council Reports and Memoranda No. 3438, October 1964

[14] M. Samimy, M. Debiasi, E. Caraballo, H. Özbay, M. Ö. Efe, X. Yuan, J. DeBonis, and J. H. Myatt, "Development of Closed-Loop Control for Cavity Flows", AIAA Paper 2003-4258, June 2003b.

[15] M. Samimy, M. Debiasi, E. Caraballo, J. Malone, J. Little, H. Özbay, M. Ö. Efe, P. Yan, X. Yuan, J. DeBonis, J. H. Myatt and R. C Camphouse, "Exploring Strategies for Closed-Loop Cavity Flow Control", AIAA Paper 2004-0576, January 2004.

[16] L. Sirovich, "Turbulence and the Dynamics of Coherent Structures", Quarterly of Applied Math, Vol. XLV, N. 3, pp. 561-590, 1987.

[17] S. Wiggins, Introduction to Applied Nonlinear Dynamical Systems and Chaos, Springer, 2003.

[18] P. Yan, M. Debiasi, X. Yuan, E. Caraballo, A. Serrani, H. Özbay, J. M. Myatt, and M. Samimy, "Modeling and Feedback Control for Subsonic Cavity Flows: A Collaborative Approach", Proc. 44th IEEE CDC-ECC, Seville, Spain, 2005.

[19] X. Yuan, E. Caraballo, P. Yan, H. Özbay, A. Serrani,J. DeBonis, J. H. Myatt and M. Samimy, "Reduced-order Model-based Feedback Controller Design for Subsonic Cavity Flows", AIAA Paper 20050293, January, 2005. 